Schwefelsãure verdampfen. Erst aus der sirupdicken Mutterlauge scheidet sich das Salz in mikroskopischen Nädelchen aus, so daß ein dicker Brei entsteht. Man befreit das Salz durch Absaugen und Aufstreichen auf Ton von der Mutterlauge. Aus wäßriger Lösung erhält man das Salz nicht zurück.

$0.9804 \mathrm{~g}$ Sbst. u. $70.4 \mathrm{~cm} 1 / 10^{-} n, \mathrm{H}_{2} \mathrm{SO}_{4}: 70.2 \mathrm{ccm}^{1 / 1} 10^{-n}$. KOH. $-0.5812 \mathrm{~g}$ Sbst.: $0.5290 \mathrm{~g} \mathrm{PbSO}_{4}$ - $1.1904 \mathrm{~g}$ Sbst.: $24.1 \mathrm{cem} 1 / 10^{-n}$. $\left.\mathrm{H}_{2} \mathrm{SO}_{4}{ }^{1}\right)$.

$\left[\mathrm{Pb}_{8}\left(\mathrm{CH}_{3}, \mathrm{CO} 0.0\right)_{4}\right]\left(\mathrm{NO}_{3}\right)_{3}+1 \mathrm{H}_{2} \mathrm{O}(1159,6)$.

Ber. $\mathrm{Pb} 62.18, \mathrm{NO}_{3} 12.40,\left(\mathrm{CH}_{3} . \mathrm{CO} .0\right) 23.62$.

Gef. \62.19, > 12.55, > 23.68 .

\title{
Bleiacetat-nitrat (VI.).
}

Dieses Salz erbält man analog dem ersten Nitrat( $\nabla$.$) beim Ter-$ hältnis von Bleiacetat zu Salpetersäure wie 2:1.

Kleine, häufig strahlenförmig angeordnete Nadeln.

Aus der wäßrigen Lösung scheidet sich das Salz nicht wieder aus.

$0.5054 \mathrm{~g}$ Sbst.: $0.4554 \mathrm{~g} \mathrm{PbSO} 4 .-2.3804 \mathrm{~g}$ Sbst.: $36.4 \mathrm{~cm} \mathrm{1/10-n.}$

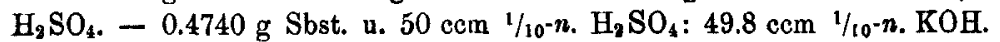

$\left[\mathrm{Pb}_{9}\left(\mathrm{CH}_{8} . \mathrm{CO} .0\right)_{3}\right] \mathrm{NO}_{3}+1 \mathrm{H}_{2} \mathrm{O}(671.4)$.

Ber. $\mathrm{Pb} 61.71, \mathrm{NO}_{8} 9.24,\left(\mathrm{CH}_{3}, \mathrm{CO} .0\right) 26.37$.

Gef. > 61.56, > 9.48,, 26.02 .

Würzburg, 28. April 1922 .

\section{Hans Stobbe und Franz Karl Steinberger: Lichtreaktionen der trans- und cis-Zimtsăuren.}

(Eingegangen am 5. Mai 1922.)

Im zweiten Hefte der diesjährigen Berichte behauptet A. W. K. de Jong ${ }^{2}$ ), daß seine ersten Untersuchungen über die Lichtreaktionen der Zimtsäuren einige Jahre früher angestellt seien, als die den gleichen Gegenstand betreffenden Arbeiten des Erstgenannten von uns. Darauf ist zu erwidern: Meine Versuche der Belichtung der cis-Zimtsäure (420) sind im Jahre 1909 begonnen. Ich habe die damaligen Präparate, wie bereits erwähnt, $\mathrm{zwei}$ Jahre hindurch beobachtet und sie dann im Sommer 1911 mit späteren, von meinem damaligen Doktoranden Jussik Pogossianz gewonnenen Belichtungsprodukten der drei isomeren cis-Zimtsäuren $\left(42^{\circ}, 58^{\circ}\right.$ und $\left.68^{\circ}\right)$ gemeinsam untersucht. Hr. Pogossianz beendete diese Arbeiten im Juli 1911, also bevor wir Kenntnis bekamen von dem im 7. Hefte des Chemischen

1) Bestimmung der Salpetersäure durch Redaktion zu Ammoniak.

ग) B. 55, $463[1922]$. 
Zentralblattes 1911, II 452 am 16. August erschienenen Referate über de Jongs Veröffentlichung in den uns damals nicht zugänglichen "Abhandlungen der Koninklijke Academie van Wetenschappen to Amsterdam * 1), natürlich auch weit vor dem Erscheinen der umfassenderen Arbeit de Jongs in den Recueil des travaux chimiques des Pays-Base 3). Die von meinem Mitarbeiter und mir erhaltenen Resultate sind dann mit allen Einzelheiten in der gedruckten Dissertation ${ }^{3)}$ des letzteren niedergelegt. Wenn ich sie erst 1919, gleichsam als vorläufige Mitteilung, in den $*$ Berichten * ${ }^{4}$ veröffentlichte, so lag dies daran, daß ich während des Krieges vier volle Jahre im Heeresdienste stand und in den Jahren 1912-1914 keine Gelegenheit gefunden hatte, die bis 1911 gewonnenen Resultate rorwiegend qualitativer Art durch die Ergebnisse der mir schon damals erforderlich scheinenden, nunmehr ausgeführten, rein quantitativen Versuche zu ergänzen. Aus diesen Darlegungen dürfte jedermann erkennen, daß Hrn. de Jongs Prioritätsanspruch unberechtigt ist. ${ }^{*}$ Hr. de Jong und ich haben unabhängig von einander und anscheinend gleichzeitig ermittelt, daß bei der Sonnenbestrablung der cis-Zimtsäuren außer der erwarteten $\beta$-Truxinsäures) auch trans-Zimtsăure und $\alpha$-Truxillsäure entstehen.

Wenn somit unsere beiderseitigen Versuchsergebnisse in dieser Hinsicht übereinstimmten, und wir auch die Bildung eines Mol. $\alpha$-Truxillsäure aus $2 \mathrm{Mol}$, trans Zimtsäure für erwiesen erachteten, waren wir betreffs der Photo-synthese der $\beta$-Truxinsäure verschiedener Meinung. De Jong glaubte annehmen zu müssen, daß 1 Mol. dieser Säure aus je $1 \mathrm{Mol}$. cis- und trans-Zimtsäure entstände, weil bei einigen Belichtungen der cis-Säure die anfänglich nur spärlich auftretende $\beta$-Truxinsäure sich erst später, nach Bildung relativ großer Mengen trans-Säure, wesentlich vermehre, und weil aus einem Gemisch von $1 \mathrm{~g}$ cis-Säure und $2 \mathrm{~g}$ trans-Säure nach vollständigem Verbrauch der ersteren $0.93 \mathrm{~g} \beta$-Truxinsäure und $1.6 \mathrm{~g} \alpha$-Truxillsäure gebildet waren.

Stobbe hielt diese Hypothese nicht für stichhaltig. Sie wäre erst bewiesen, wenn bei dem eben genannten Versuche die ursprünglich vorhandene cis-Säure von der gebildeten $\beta$-Truxinsäure an Gewicht übertroffen worden wäre, und selbst in diesem Falle noch nicht uneingeschränkt, da ja auch nach Störmers ${ }^{6}$ ) jüngsten Berichten bei

1) Koninkl. Akad. 20, 55 [1911].

3) Pogossianz, Leipzig 1912.

2) R. 31, 258 [1912].

4) B. 52, 666 [1919].

5) Über die Nomenklatur vergl. Stŏrmer, B. 54, 78 [1921]; 55, 1871 [1922].

6) Mit Laage, B. 64, 79 [1921]. 
Belichtung fester trans-Säure - nach vorausgegangener partieller Isomerisierung zur cis-Säure - etwas $\beta$-Truxinsäure aufträte. Es gelte also das Schema:

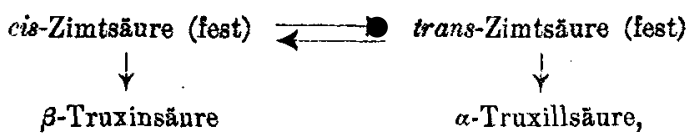

das gegen das früher entworfene ${ }^{1}$ ) nur durch den von rechts nach links führenden Pfeil ergänzt ist. Bei der direkten Bildung der $\beta$-Truxinsäure sei daher die cis-Säure allein beteiligt, ebenso wie die trans-Säure für sich allein zu $\alpha$-Truxillsäure polymerisiert werde.

Um diese Frage zu entscheiden, haben wir im Sommer 1921 die vorliegenden Versuche unternonmen. Ausgangsmaterial war $\mathbf{K a h l}$ baumsche trans-Zimtsäure $\left(133^{\circ}\right)$ and allo-Zimtsäure $\left(68^{\circ}\right)$, die teils durch Halbreduktion von Phenyl-propiolsäure nach $\mathrm{Paal}$ und Hartmann ${ }^{2}$ ), teils auf photochemischem Wege nach Störmer ${ }^{3}$ ) dargestellt war. Da die Intensität des Sonnenlichtes wechselt, baben wir für unsere ersten Versuche gemäß dem früher ${ }^{4}$ ) entwickelten Arbeitsprogramm zunächst nur das möglichst konstante Licht der Quarz-Quecksilberlampe benutzt. Besonders wurde darauf geachtet, $\mathrm{da} B$ die Objekte während der Belichtung ihren Aggregatzustand nicht änderten, also sich entweder dauernd in flüssigem oder dauernd in festem Zustande befanden.

\section{Das Verbalten flüssiger allo-Zimtsäure-Schmelze im Licht.}

Hier zeigte sich, daß auch bei langer, 96-stündiger Belichtung an der Quarzlampe zwar eine weitgehende Is omerisierung zur transSäure eintrat, daß aber weder Truxill- noch Truxinsäuren gebildet waren. Die Schmelzen verhalten sich demnach im Lichte ebenso wie die benzolischen Lösungen ${ }^{5}$ ). Man kann also verallgemeinernd sagen, daß beim Belichten von Zimtsäuren im flüssigen Zustande, in Lösung oder in Schmelze, nur Isomerisierung, aber keine Polymerisation erfolgt.

Über die Beteiligung der einzelnen Strahlengebiete an diesem Isomerisierungsvorgange gibt Aulschluß eine Versuchsreihe, bei der " $/ 4$-benzolische trans-Zimtsäure-Lösungen in drei Rohren gleichzeitig bestrahlt wurden.

1) 1. c. S. 668 .

8) B. 42,4869 [1909].

5) Störmer, B. 42, 4865 [1909]. - Für methylalkoholische Lösung,

2) B. 42, 3930 [1909].

) B. $52,670[1919]$. B. 47,1806 [1914]. 
Quarzrohr, $24 \mathrm{~mm}$ Durchmesser, durchlăssig für alle Strablen bis zur Absorptionsgrenze des Benzols,

Uviol-Glasrohr, $24 \mathrm{~mm}$ Durchmesser, durchlässig bis $271 \mu \mu$,

Gewöhnliches Glasrohr, $23 \mathrm{~mm}$ Durchmesser, durchlässig bis $320 \mu \mu$.

Lampenabstand $8 \mathrm{~cm}$.

Stromstärke $4.4 \mathrm{Amp}$.

\begin{tabular}{c|c|c|c}
\hline $\begin{array}{c}\text { Belichtungs- } \\
\text { stunden }\end{array}$ & \multicolumn{3}{|c}{$\begin{array}{c}\text { Prozentgehalt an cis-Säure in } \\
\text { Uviol- }\end{array}$} \\
& Quarz- & gew. Glas \\
\hline & & & \\
62.3 & 9.0 & 6.3 & 3.2 \\
92.5 & 22 & 16.6 & 4.8 \\
137.5 & 23.5 & 18.8 & 5.1 \\
& & & 6.0
\end{tabular}

Man ersieht hieraus, daß den Strablen von $270-320 \mu \mu$ die Hauptwirkung zuzuschreiben ist, dababer auch diekürzerwelligen unterbalb $270 \mu \mu$, sowie auch die längerwelligen oberhalb $320 \mu \mu$ nicht ohne Einfluß sind. Isomerisieren hier die letzteren in 137.5 Belich-

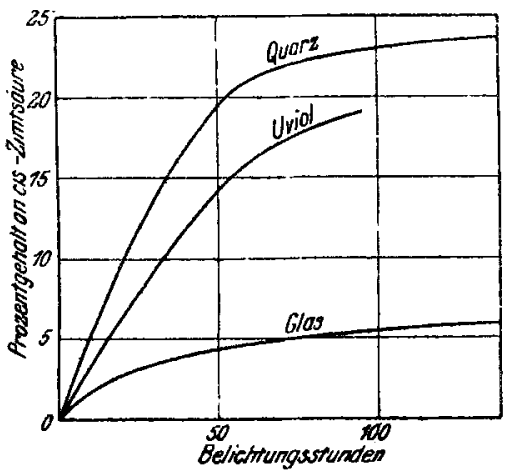
tungsstunden nur $6 \%$ der transSäure, so lieferte ein Nebenversuch, bei dem eine benzolische trans-Säurelösung zwei Monate in einer dicken Glasflasche an der Sonne belichtet wurde, $16 \%$ cis-Säure. Der im Quarzrohr erreichte Wert von $23.5 \%$ cisSäure entspricht übrigens sehr annähernd den bei analogen Versuchen Störmers') gefundenen Werten für das Gleichgewicht der beiden Isomeren.

\section{Belichtungsversuche mit Zimtsäuren \\ in festem $\mathrm{Zustande.}$}

Versuchsobjekte waren einheitliche allo-Zimtsäure, einheitliche käufliche trans-Zimtsäure, sowie Gemenge gleicher Gewichtsteile von beiden Isomeren, deren Pulver entweder trocken gemischt oder vorher miteinander verschmolzen waren. Als Lichtquelle diente zuerst die Quarz-Quecksilberlampe, dann aber auch aus später einzusehenden Gründen das Sonnenlicht.

1) B. 42, 4869 [1909]. 
Nach de Jong ${ }^{1}$ ), Störmer und Laage ${ }^{2}$ ) entstehen beim Belichten von trans-Zimtsäure neben $\alpha$-Truxillsäure noch geringe Mengen anderer isomerer Truxill- und Truxinsäuren. Da mit dem Auftreten solcher Nebenprodukte auch bei der Belichtung der allo-Zimtsäure zu rechnen war, andererseits es aber hier nur auf das Gewichtsverbältnis von $\alpha$-Truxillsäure, $\beta$-Truxiasäure, cis- und trans-Zimtsäure ankam, haben wir bei der Aufarbeitung der Reaktionsprodukte geringe Mengen anderer isomerer Säuren nicht berücksichtigt.

Die in den großen Rubriken (1, 2, 3 usw.) vereinigten Versuche sind immer gleichzeitig, also unter genau gleichen Bedingungen ausgeführt.

I. Versuchean der Quarz-Quecksilberlampe.

\begin{tabular}{c|c|c|c|c|c|c}
\hline & & & \\
\hline
\end{tabular}

II. Versuche im Sonnenlicht.

Versuchsobjekte, $z$ wischen $z$ wei Glasplatten, blieben während der Belichtung fest und fast rein weiß. Bei der wenig konstanten Licht1) C. 1919, III $1000 . \quad$ ?) B. 54, 80 [1921]. 
quelle sind genau übereinstimmende Resultate natürlich nicht zu erwarten.

\begin{tabular}{|c|c|c|c|c|c|c|c|}
\hline 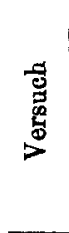 & Versachsobjekst & 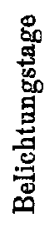 & 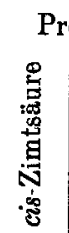 & ozents & 禀。 & an & Bemerkungen \\
\hline $\begin{array}{l}\text { 1. a) } \\
\text { b) }\end{array}$ & $\begin{array}{c}5 \mathrm{~g} \text { allo-Zimtsäure, } \\
\text { pulverisiert . . }\end{array}$ & $\begin{array}{l}14 \\
28\end{array}$ & $\begin{array}{l}44.6 \\
-\end{array}$ & $\begin{array}{l}37.1 \\
22.0\end{array}$ & $\overline{20.1}$ & $\begin{array}{l}18.3 \\
50.9\end{array}$ & $\begin{array}{l}\text { meist trübes Wetter; } \\
\text { die letzten } 14 \text { Tage } \\
\text { sonnig }\end{array}$ \\
\hline b) & $\begin{array}{l}1 \mathrm{~g} \text { allo-Säure, pul- } \\
\text { verisiert. } . . \\
1 / 2 \text { g allo- und } 1 / 2 \mathrm{~g} \\
\text { trans-Säure, pulve- } \\
\text { risiert. . . . . }\end{array}$ & 17 & 14.8 & 39.2 & 14.3 & 31.7 & \\
\hline $\begin{array}{l}\text { 3. a) } \\
\text { b) }\end{array}$ & $\begin{array}{l}1 \mathrm{~g} \text { allo-Säure, er- } \\
\text { starrte Schmelze. } \\
1 / 2 \mathrm{~g} \text { allo- und } 1 / 2 \mathrm{~g} \\
\text { trans - Säure, er- } \\
\text { starrte Schmelze. }\end{array}$ & 20 & $\begin{array}{l}12.3 \\
12.7\end{array}$ & 39.3 & 21.1 & 17.9 & \\
\hline $\begin{array}{l}\text { 4. a) } \\
\text { b) }\end{array}$ & $\begin{array}{l}1.5 \mathrm{~g} \text { allo-Säure, er- } \\
\text { starrte Schmelze . } \\
1 \mathrm{~g} \text { allo- und } 1 \mathrm{~g} \\
\text { trans - Särre, er- } \\
\text { starrte Schmelze. }\end{array}$ & 27 & 8.1 & 48.7 & 30.3 & 12.9 & \\
\hline b) & $\begin{array}{l}1.5 \mathrm{~g} \text { allo-Säure, } \\
\text { pulverisiert. . } \\
1 \mathrm{~g} \text { allo- und } 1 \mathrm{~g} \\
\text { trans-Säure, pulve- } \\
\text { risiert. . . . . }\end{array}$ & 27 & 6.4 & 34.6 & 56.4 & 50.3 & \\
\hline 6. & $\begin{array}{c}3 \mathrm{~g} \text { trans-Sãure, pul- } \\
\text { verisiert } .\end{array}$ & 23 & - & 14.3 & 85.7 & - & $\begin{array}{l}\text { geringfügige Neben- } \\
\text { produkte; } \beta \text {-Truxin- } \\
\text { săure vernachlăssigt }\end{array}$ \\
\hline
\end{tabular}

Bei einem Vergleich der Lampen- und Sonnenlichtversuche sind die Isomerisierungs- und Polymerisierungsreaktionen wieder gesondert zu betrachten. Die Isomerisierung (cis-Säure $\rightarrow$ trans-Säure) verläuft in beiden Fällen ähnlich (Versuch $I, 1$ und II, 1). Die Polymerisierung $\left(2 \mathrm{C}_{8} \mathrm{H}_{8} \mathrm{O}_{2} \rightarrow\left[\mathrm{C}_{8} \mathrm{H}_{8} \mathrm{O}_{9}\right]_{2}\right)$ führt dagegen zu gewaltigen Onterschieden in den Ausbeuten an Dimeren. Rechnet man jeden Belichtungstag der Versuche unter II. zu etwa 7 Sonnenlioht-Stunden, so ergibt sich: 
$\alpha-\operatorname{Tr} . \quad \beta-T r . \quad \begin{gathered}\text { Dimere } \\ \text { (totsl) }\end{gathered}$

czs-Säure, 200 Stdu. Lampenlicht $(I, 1)$. . . . - - $4.4 \%$

- , $196 \quad$ Sonnenlicht (II, 1) . . . $20.1 \% \quad 50.9 \% \quad 71.0 \%$

trans.Säure, 364 Stdn. Lampenlicht $(\mathrm{I}, 4)$. . . . $2 \%$

$>, 161$ Sonnenlicht $($ II, 6) . . . 85.7\% - 85.7\%

Diese Verschiedenheiten sind begründet durch die Ungleichheit des zur Wirkung gelangten Lichtes. Langwellige U-V.Strahlen begünstigen die Polymerisation. Kurzwellige U-V-Strahlen verhindern die Polymerisation; sie wirken, wie demnächst eingehend gezeigt werden wird, depolymerisierend auf die Truxill- und Truxinsäuren. Schon Störmer und Förster ${ }^{1}$ ) berichten über derartige Vorgänge bei der Dltraviolett-Bestrahlung von Natriumsalzen der beiden Dimeren.

Von unseren in Leipzig ausgeführten Sonnenlicht-Versuchen beziehen sich sechs (II. 1a, 1b, 2a, 3a, 4a und 5a) aul einheitliche cis-Säure. Wertet man die Ergebnisse anderer Belichtungen derselben Säure in entsprechender Weise aus, so erhält man folgendes Bild:

\begin{tabular}{|c|c|c|c|c|c|}
\hline & \multicolumn{4}{|c|}{ Prozentgehalt an } \\
\hline & & trans-S. & $c i s-\mathrm{S}$. & $\alpha$-Truxills. & $\beta$-Truxins \\
\hline & $11 / 4$ Stdn. & $?$ & $?$ & $?$ & 5.5 \\
\hline & $\begin{array}{r}2 \\
19\end{array}$ & ? & $?$ & ? & 7.8 \\
\hline $\begin{array}{l}\text { de Jong } \\
\text { (Buiten- }\end{array}$ & $\begin{array}{c}\text { unter Glas } \\
19 \mathrm{Stdln} .\end{array}$ & vorbanden & $?$ & - & 56.6 \\
\hline $\begin{array}{l}\text { zorg), } \\
\text { ( } 55,466\end{array}$ & ohne Glas & vorhanden & $i$ & - & 68.4 \\
\hline $0.00,700$ & $5 \mathrm{Stdn}$. & vorhanden & $?$ & $?$ & 43.6 \\
\hline & 5 Stdn. & vorhanden & ? & 2 & 67.2 \\
\hline $\begin{array}{l}\text { de Jong } \\
\text { (Buiten- }\end{array}$ & $\begin{array}{c}6 \text { Tage, nur } \\
\text { vormittags } \\
\text { obne Zeitangabe }\end{array}$ & $\begin{array}{l}36.2 \\
30.0\end{array}$ & 56.5 & $4 \overline{9.0}$ & $\begin{array}{r}7.3 \\
21.0\end{array}$ \\
\hline $\begin{array}{l}\text { zorg), } \\
\text { R. 31,258 }\end{array}$ & 17 Tage, nur & 32.5 & - & 40.0 & 27.5 \\
\hline [1912] & vormittags & 21.2 & - & 10.6 & 68.1 \\
\hline $\begin{array}{l}\text { St örmer } \\
\text { (Rostock), }\end{array}$ & $\begin{array}{l}\text { 1. } 8 .-1.10 \\
\text { 8. } 7 .-1.10 .\end{array}$ & $\begin{array}{c}\text { wenig } \\
\text { ? }\end{array}$ & $\begin{array}{l}? \\
?\end{array}$ & $\stackrel{?}{14.9}$ & $\begin{array}{l}21.7 \\
36.1\end{array}$ \\
\hline 1262 & Hochsommer & Spuren & vorhanden & - & 65.0 \\
\hline
\end{tabular}

Diese an verschiedenen Orten und unter ungleichen Lichtverhältnissen ausgeführten Versuche lebren, daß die cis-Säure immer und meist überwiegend $\beta$-Truxinsäure liefert, daß aber auch, und zwar bei längerer Belichtung, die Isomerisierung zur trans-Säure mit nachfolgender Polymerisierung zur $\alpha$-Truxillsäure erfolgt.

1) B. $\check{2} 2,1263[1919]$. 
Für die gewöhnliche, nicht direkt vorher umkrystallisierte transZimtsäure liegen außer unserem obigen Versuche II, 6 noch einige andere, mebr oder weniger quantitativ durchgeführte Sonnenlichtversuche vor.

\begin{tabular}{|c|c|c|c|c|c|c|}
\hline \multirow{5}{*}{$\begin{array}{c}\text { de Jong } \\
\text { (Buiten- } \\
\text { zorg), } \\
\text { B. } 55,468\end{array}$} & & trans-S. & $c i s-\mathrm{S}$. & $a$-Truxills. & $\beta$-Truxins & \\
\hline & $\begin{array}{l}5 \mathrm{Stdn} \\
\text { unter Glas }\end{array}$ & $?$ & ? & 26.0 & - & \\
\hline & unter Glas & $?$ & : & 36.4 & $\cdots$ & \\
\hline & $\begin{array}{l}\text { ohne Glas } \\
5 \text { Stdn. }\end{array}$ & $?$ & ? & 65.0 & - & \\
\hline & onne Glas & $?$ & $?$ & 70.5 & - & \\
\hline $\begin{array}{c}\text { Riiber, } \\
\text { B. 35, } \\
2908\end{array}$ & 50 Stdn. & $?$ & ? & 70.0 & - & \\
\hline $\begin{array}{l}\text { Störmer, } \\
\text { B. } \mathbf{5 4}, 80\end{array}$ & ohne Zeitangabe & 33.91 & - & 65.01 & 0.97 & $\begin{array}{c}0.11 \\
\varepsilon-\text { Truxins. }\end{array}$ \\
\hline $\begin{array}{l}\text { Stobbe } \\
\text { und } \\
\text { Lehfeldt }\end{array}$ & $\left\{\begin{array}{l}\text { 2. 7. }-1.10 .20 \\
2 \text { Monate } \\
21.5 .-23.10 .20\end{array}\right.$ & $\begin{array}{l}10.00 \\
32.63 \\
9.8\end{array}$ & - & $\begin{array}{l}53.70 \\
5.08 \\
62.5\end{array}$ & $\begin{array}{l}6.90 \\
0.50 \\
7.5\end{array}$ & $\begin{array}{l}29.4 \text { Harz } \\
10.87 \% \\
20.2 \%\end{array}$ \\
\hline
\end{tabular}

Man erkennt, daß hier die Verbältnisse ganz anders liegen wie bei der cis-Säure. Die trans-Sãure wird fast ausschlieBlich zu $\alpha$-Truxillsäure polymerisiert. Erst bei längerer Belichtungszeit traten neben einer recht starken Verharzung verhältnismäßig geringe Mengen $\beta$-Truxinsäure auf. cis-Säure ist nicht aufgefunden und konnte auch schwerlich erwartet werden, da sie ja bei der langen Belichtung in $\beta$-Truxinsäure verwandelt werden mußte.

$\mathrm{Zu}$ welchen Ergebnissen haben nun die Belichtungen der Zimtsăure-Gemische geführt? Hierfür sind maßgebend die Parallelversuche I, $1 \mathrm{~b}$ (Quarzlampe) sowie II, $2 \mathrm{~b}, 3 \mathrm{~b}, 4 \mathrm{~b}$ und $5 \mathrm{~b}$ (Sonnenlichf). Sie zeigen, daß ein zur Hälfte aus cis- und trans-Säure bestehendes Gemenge prozentual immer nur etwa halb soviel $\beta$-Truxinsāure produziert als ein aus cis-Säure allein bestehendes Versuchsobjekt. Hieraus ergibt sich, dab die der cis-Säure beigemengte transSäure keine Vermehrung der $\beta$-Truxingäure bewirkt hat, und daB also, entgegen der deJongschen Voraussetzung, ein Mol. $\beta$-Truxinsäure allein aus 2 Mol. cis-Säure gebildet. wird.

Von den sechs für Truxinsäuren möglichen Raumformeln ${ }^{1}$ ) kann die $\beta$-Säure nach de Jong und nach Störmer und Förster ${ }^{2}$ ) nur eine der folgenden mit cis-Stellung der Carboxyle haben; sie bildet leicht ein inneres Anhydrid und wird durch Kalisobmelze in
ग) Numerierung nach B. 54, 86 [1921].
ग) B. 52,1255 [1919]. 
eine trans-Form, die $\delta$-Truxinsüure, umgelagert. De Jong entscheidet sich für die Formel III, weil nur diese allein seiner Hypothese von der Entstehung der $\beta$-Săure aus je $1 \mathrm{Mol}$, cis- und transSänre gerecht wird. Störmer und Scholtz ${ }^{1}$ ) machen dagegen geltend, daß die Racemformel III für die leicht spaltbare $\zeta$-Truxinsäure zu reservieren sei und nur die Mesoformeln I oder II für die $\beta$-Säure in Betracht kommen können.

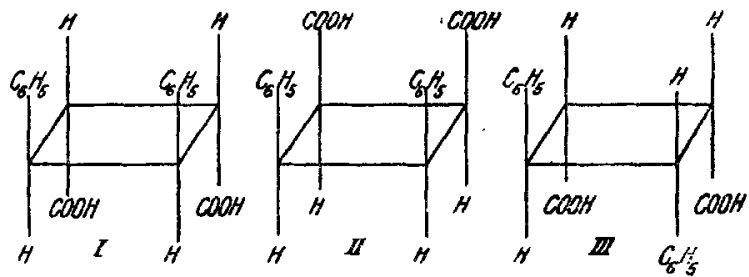

Man erkennt also, wie Störmer und wir aus ganz verschiedenen Gründen die Hypothese de Jongs ablehnen müssen. Ob Formel I oder II der $\beta$ Säure zukommt, ist noch nicbt entschieden. Nach Störmer sei I wahrscheinlicher, weil die $\beta$-Säure kein Truxon bilde, und weil eine Säure mit vier, auf einer Seite der Ringebene liegenden Gruppen ein ziemlich labiles Gebilde sein dürftex, und demnach dem Charakter der $\beta$-Säure nicht entspräche.

Nach Versuchen von Stobbe und Alice Lehfeldt werden $\beta$-Arylacrylsäuren und unter ihnen auch trans-Zimtsãure nicht nur in trockner Form, sondern auch in wäßriger Suspension dimerisiert und zu Benzaldehyd autoxydiert. Beide Vorgänge werden beschleunigt durch Zusatz einiger Tropfen Salzsäure, die Autoxydation mehr als die Dimerisation. Die Folge davon ist, daß bei langer Belichtung die Ausbeute an $\alpha$-Truxillsäure sinkt und die Menge des Benzaldehyds steigt.

Om einen weiteren Einblick in die Lichtreaktionen der isomeren Zimtsäuren zu gewinnen, haben wir zunächst einige analoge Versuche mit cis-Säure $\left(68^{\circ}\right)$ im Sonnenlicht angestellt und durch Prüfung der Schmelzpunkte verfolgt.

\begin{tabular}{|c|c|c|c|c|c|}
\hline \multicolumn{6}{|c|}{ Versuch 1 . } \\
\hline & & & & $\begin{array}{l}\text { in } \\
\text { Wasser }\end{array}$ & $\begin{array}{l}\text { Suspensionen } \\
\text { in salzsäure- } \\
\text { haltigem Wasser }\end{array}$ \\
\hline $\begin{array}{l}\text { Nach } \\
?\end{array}$ & $\begin{array}{l}2 \mathrm{t} \\
6 \\
10\end{array}$ & 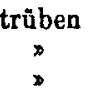 & $\begin{array}{c}\text { Tagen } \\
?\end{array}$ & $\begin{array}{r}64-75^{\circ} \\
126-140^{\circ} \\
130-190^{\circ}\end{array}$ & $\begin{array}{r}64-90^{\circ} \\
126-165^{\circ} \\
130-190^{3}\end{array}$ \\
\hline \multicolumn{6}{|c|}{ Versuch II. } \\
\hline$\underset{D}{N a c h}$ & & nnigen & $\begin{array}{l}\text { Tag } \\
\text { Tagen }\end{array}$ & $\begin{array}{r}64-85^{\circ} \\
100-125^{\circ}\end{array}$ & $\begin{array}{r}66-90^{\circ} \\
120-145^{\circ}\end{array}$ \\
\hline
\end{tabular}

1) B. 54, 87 [1921]. 
Da die Belichtungsprodukte reichliche Mengen $\beta$-Truxinsäure und Benzaldehyd enthielten, sind also die Photoreaktionen der cisSäure ganz ähnlich wie bei der trans-Säure verlaulen. Auch hier wird, besonders in Gegenwart von Salzsăure, bei längerer Belichtung die Endausbeute an $\beta$-Truxinsäure wegen der starken Antoxydation der cis-Säure wesentlich verringert.

Diese Ergebnisse ließen ratsam erscheinen, Parallelbelichtungen von einbeitlicher cis-Säure und von Gemischen voncisund $\operatorname{trans} s$-Säure auszuführen.

Versuchsobjekte in salzsaurer Suspension. - Gleich große Glaskolben. Sonnenlicht.

\begin{tabular}{|c|c|c|c|c|c|c|c|}
\hline \multirow{2}{*}{$\begin{array}{l}\text { Ver- } \\
\text { such }\end{array}$} & & \multirow{2}{*}{$\begin{array}{l}\text { Be- } \\
\text { lich- } \\
\text { tungs- } \\
\text { tage }\end{array}$} & \multicolumn{5}{|c|}{ Prozentgehalt des Reaktionsprodukts } \\
\hline & & & $\begin{array}{l}\text { cis- } \\
\text { Säure }\end{array}$ & $\begin{array}{l}\text { trans- } \\
\text { Sãure }\end{array}$ & $\begin{array}{c}\alpha \text {-Truxill- } \\
\text { süure }\end{array}$ & $\begin{array}{c}\beta \text {-Truxin- } \\
\text { säure }\end{array}$ & Harz \\
\hline 1 & $\begin{array}{r}1 \mathrm{~g} \text { allo-Säure } \\
1 / 2 \mathrm{~g} \text { allo- und } \\
1 / 2 \mathrm{~g} \text { trans-Säure }\end{array}$ & $\left\{\begin{array}{l}9 \\
9\end{array}\right.$ & - & $\begin{array}{l}53.9 \\
58.4\end{array}$ & $\begin{array}{r}- \\
18.1\end{array}$ & $\begin{array}{l}38.2 \\
18.4\end{array}$ & $\begin{array}{l}7.9 \\
5.1\end{array}$ \\
\hline 2 & $\begin{array}{l}1 \mathrm{~g} \text { allo-Säure } \\
1 / 2 \mathrm{~g} \text { allo- und } \\
1 / 2 \mathrm{~g} \text { trans-Säure }\end{array}$ & $\left\{\begin{array}{l}20 \\
20\end{array}\right.$ & - & $\begin{array}{l}53.53 \\
55.6\end{array}$ & $\begin{array}{l}0.33 \\
21.7\end{array}$ & $\begin{array}{l}38.13 \\
15.7\end{array}$ & $\begin{array}{l}8.01 \\
7.0\end{array}$ \\
\hline
\end{tabular}

Bei allen Versuchen starker Benzaldehyd-Geruch und recht beträchtliche Harzbildung. Im übrigen zeigt sich auch hier, daß $1 \mathrm{~g}$ einheitliche cis-Säure ungefähr doppelt soviel $\beta$-Truxinsăure produziert, als ein Gemenge von $1 / 2 \mathrm{~g}$ cis- und 1/2 $\mathrm{g}$ trans-Säure in gleichem Zeitraum. Die Bildung der $\beta$-Truxinsäure ist also auch hier, entgegen der de Jongschen Hypothese unabhängig von der Gegenwart der beigemengten trans-Säure. Beide Isomeren polymerisieren sich, auch in Suspension selbständig zu ibren Dimeren.

Auffällig erscheint nur, daß die während des Belichtungsvorganges durch Isomerisierung frisch gebildete trans-Sāure viel langsamer polymerisiert wird, als die in Pulverform beigemengte Săure. Dieses Phänomen erklärt sich durch die fortschreitende Änderung des Absorptionsvermögens der belichteten Krystalle. Zunächst umzieht sich unter der Wirkung der langwelligen U-V-Strahlen die Oberfläche der cis-Säure-Krystalle mit einer Scbicht von $\beta$-Truxinsäure. $\mathrm{Da}$ diese weit lichtdurchlässiger ${ }^{1}$ ) als die cis-Säure ist, werden nicht nur langwellige, sondern auch kurzwellige U.V.Strahlen in das

1) B. 52, 1027 [1919]. 
Krystallinnere eindringen und dort die Isomerisation zur trans-Säure uad nachfolgend die Polymerisation zur $\alpha$-Truxillsäure hervorrufen. Wird dann die veränderte Oberflächenschicht der Krystalle dicker, so tritt immer weniger Licht in die nur noch schwach korrodierten Krystalle ein. Die Photoreaktionen kommen zum Stillstand, so daB also die trans-Säure, gleichsam eingekapselt in die Säuren, der weiteren Polymerisation entzogen wird.

In seiner eingangs zitierten Abhandlung teilt de Jong mit, daß die zuerst von Lehmann ${ }^{1}$ ) erkannte nadelförmige, metastabile Form der trans-Zimtsäure ( $\beta$-Form) darch Sonnen-Bestrahlung leicht in die $\beta$-Truxinsäure übergehe, während, wie oben gezeigt, die gewöhnliche, rautenförmige, stabile trans-Zimtsäure ( $\alpha$-Form) vornehmlich nur zu $\alpha$-Truxillsäure polymerisiert wird. Wir stehen also vor der sehr beachtenswerten Tatsache, daß diese beiden transZimtsäuren in festem Zustande photochemisch verschieden reagieren. Sie liefern verschiedene Derivate und wären daher als zwei chemisch isomere $\beta$-Pbenyl-acrylsäuren anzusprechen, vergleichbar mit der Malein- und Fumarsäure, die ja beispielsweise durch gelinde Oxydation $\mathrm{zwei}$ verschiedene Weinsäuren und mit Brom $\mathrm{zwej}$ verschiedene Dibrom-bernsteinsäuren bilden. Auch lagern sich die Zwillinge der beiden genannten Säurepaare wechselseitig ineinander um, allerdings mit dem Unterschiede, daß diese Vorgänge bei den Äthylendicarbonsăuren meist viel langsamer, als bei den $\alpha$ - und $\beta$-Zimtsäuren erfolgen und außerdem auch nicht durch Impfkrystalle beeinflußt werden.

Ist die $\beta$-Zimtsäure auf einem der vielen, von de Jong und früheren Forschern vorgeschlagenen Wegen erzeugt und setzt die Belichtung bald nach der Krystallisation ein, so wird verhältnismäßig mehr $\beta$-Truxinsäure gebildet, als wenn die Belichtung erst später nach partieller Umwandlung in die $\alpha$-Zimtsäure begonnen hätte. Hierfür als Belege zum ersten die oben von uns beschriebenen Versuche mit salzsauren Suspensionen, bei denen die allo-Zimtsäure zur $\beta$-Truxinsäure, die käufliche $\alpha$-Zimtsäure zur $\alpha$-Truxillsäure polymerisiert wurde; zum zweiten die von Alice Lehfeldt beobachtete Erscheinung, bei der frisch aus ihrer Natriumsalz-Lösung gefällte und belichtete Zimtsäure zu unserer großen Überraschung nur $\beta$-Truxinsäure und keine $\alpha$-Truxillsäure geliefert hatte.

$\mathrm{Je}^{2} 20 \mathrm{~g}$ Sảure, in salzsaurer Suspension, 5 Monate im Sonnenlicht.

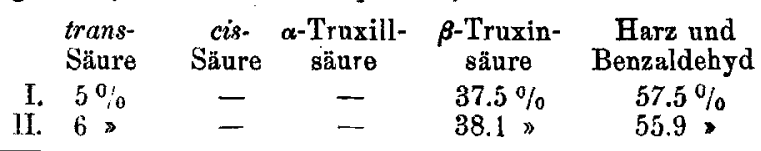

1) Z. Kr. 10, 329 [1885].

Berichte d. D. Chem. Gesellschaft. Jahrg. LV. 
Da nach de Jong aus einer Lösung des zimtsauren Natriums die $\beta$-Zimtsäure gefällt wird, dürfte bei unseren Versuchen wenigstens zu Anfang nur diese Säure belichtet worden sein.

Ohne den spezifisehen Unterschieden der $\alpha$ - und $\beta$-Zimtsäure Rechnung zu tragen, schlieBt de Jong (loc. cit. S. 466 suba), daß »die trans Säure $\alpha$ - und $\beta$-Truxillsäure geben kavn«. Dieser Satz ist in solcher Verallgemeinerung nicht zutreffend; ebenso wenig wie der zweite Sałz (sub b), nach dem »das Entstehen von $\beta$-Truxillsäure aus allo-Zimtsäure nicht direkt, sondern indirekt über die trans-Säure vor sich gehte. Eine solche Deutung dieses Vorganges würde allen bisherigen Erfahrungen widersprechen. Wäre nicht viel wahrscheinlicher die umgekehrte Annahme, nach der die sehr reaktive $\beta$-Zimtsäure (schneller als die $\alpha$-Zimtsäure) zur allo-Zimtsäure isomerisiert und diese dann normalerweise zur $\beta$-Truxinsäure polymerisiert werde? Hiernach würde sich dann das ganze Probiem auf die Frage nach der ungleichen Geschwindigkeit der Photo-isomerisierung der beiden trans-Zimtsäuren zur cis-Säure zuspitzen. Für eine Erörterung dieser Frage muß man vor allem gegenwärtig halten, daß die hier vorliegenden Photoreaktionen nicht in Lösungen oder Schmelzflüssen, sondern in Krystallen vor sich gehen. Träfe nämlich das erstere zu, dann würde natürlich eine solche Geschwindigkeitsdifferenz in isotropen Medien für eine chemische Verschiedenheit, d. h. für die chemische Isomerie der $\alpha$ - und $\beta$-Zimtsäuren sprechen. Ganz anders in krystallisierten Gebilden. Wir wissen, daß die Lichtdurchlässigkeit und die Lichtabsorption anisotroper Krystalle je nach der Acbsenrichtung verschieden sind, und $\mathrm{da} B$ demnach auch die Geschwindigkeit der Lichtreaktionen auf den ungleichen Flächen eines solchen Krystalls verschieden sein wird. Da nun nach Lehmann die $\alpha$ - und die $\beta$-Zimtsäure sehr verschiedene Krystallausbildung haben, könnte auch hiervon das sehr abweichende photochemische Verhalten der beiden Säuren abhängen. $\alpha$ - und $\beta$-Zimtsäure brauchten also nach unserer Interpretation ihrer Lichtvorgänge nicht chemisch isomer zu sein, sondern könnten ebenso gut als zwei Formen einer dimorphen Zimtsäure angesprochen werden ${ }^{1}$ ).

1) Äbnlich liegen die Verhältnisse bei anderen Verbindungen, z. B. beim $\beta$-Tetrachlor-a-keto-naphthalin (Schmp. 115.50):

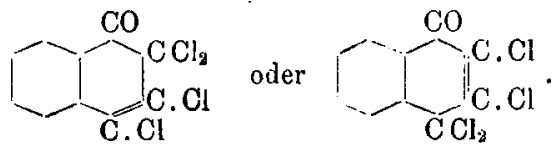

Seine aus Lösungen abgeschiedenen rhombischen Krystalle sird stark phototrop: 
Zur experimentellen Prüfung dieser beiklen Frage sind natürlich sehr viele, bereits begonnene Einzelversuche erforderlich, bei denen aber nicht, wie in den letzten Berichten von de Jong nur die Ausbeuten an den beiden Polymeren, sondern, wie früher üblich, auch der Gehalt der Belichtungsprodukte an monomeren Säuren berücksichtigt werden wird. Diese in der letzten Abhandlung de Jongs vorhandene Lücke muß unbedingt ausgefüllt werden.

Werden somit die beiden ersten Sätze de Jongs (sub a und b) beanstandet, so gilt dies auch von seinem dritten Satze (sub c), nach dem er seine frübere 1912 geäußerte Annahme - Entstehung von 1 Mol. $\beta$-Truxinsäure aus je $1 \mathrm{Mol}$. trans- und allo-Zimtsäure - selbst widerlegt habe. Diese Widerlegung ist erst durch unsere Arbeiten erfolgt.

$$
\text { farblos } \underset{\text { Dunkelheit }}{\stackrel{\text { Licht }}{\gtrless}} \text { Violett, }
$$

während die durch längeres Erwärmen auf $100^{\circ}$ verwitterten Krystalle, ebenso wie ihre wieder erstarrten Schmelzen diese Eigenschaiten vollkommen verloren haben (Marckwald, Ph. Ch. 30, 143 [1899]; vergl. A. 359, 36 [1908]). Wir haben also zwei Formen des Ketons, eine licht-empfindliche and eine licht-stabile, von denen aber die Ietztere durch bloßes Umkrystallisieren wieder in die erste phototrope Form tibergeführt werden kann.

Durch Kondensation von Fluorenon und Bernsteinsäure-ester entstehen Fulgensäuren, die bei der Anhydrisierung drei prächtig krystallisierende, chemisch verschiedene $B$ is-diphenglen-fnlgide (I.) liefern, das rote alloFulgid, Schmp. $244^{\circ}(\mathrm{A} \alpha)$, das gelbe allo-Fulgid, Schmp. $244^{\circ}$ (A $\beta$ ) und das grünschwarz erscheinende, in Wahrheit aber purpurne, kantharidenglänzende Fulgid, Schmp. $209^{\circ}$ (B).

I.

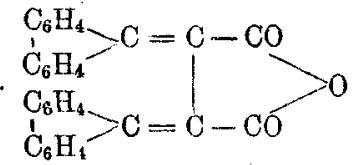

II.

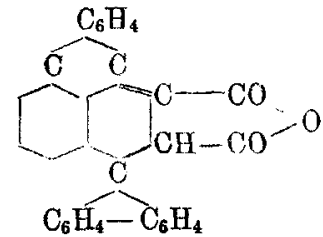

Die Krystalle der beiden allo-Fulgide sind lichtstabil, die Krystalle des purpurnen Fulgids B werden dureh Bestrablung mit Sonnenlieht in wenigen Stunden in ein gelbes, isomeres Photo-anhydrid ron Dihydro-naphthalin-Konstitution (II.) umgelagert (vergl. Jüntgen, Dissertat., Leipzig 1908, 21; Dietzel, Dissertat, Leipzig 1921, 93). Bei Belichtung ihrer Lösungen, beispielsweise in Aceton, liefern alle drei Falgide das obige Photo-anhydrid; $A \alpha$ und $A \beta$ jedoch nar nach intermediärer Umlagerung in $B$. Ich werde demnächst hierüber im Zusammenhange mit der Photochemie anderer Fulgide berichten.

Stobbe. 
Schließlich bebandelt de Jong in einem besonderen Abschnitte noch den Einflub der Temperatur auf das Entsteben der $\alpha$-Truxillsäure beim Belichten der allo-Zimtsäure*. Er meint, da $B$ die Temperatursteigerung allein die Ausbeute an $\alpha$-Truxillsäure erhöhe. Hiergegen ist nochmals $z u$ betonen, daß nicht die böhere Temperatur allein, sondern vornehmlich das Flüssigwerden der allo-Zimtsüure bei Temperaturen über $60^{\circ}$ makgebend ist. In der allo-Zimtsäure-Schmelze findet, wie oben eingehend dargetan, eine sebr leichte Isomerisierung zur trans-Säure statt. Und wird dann der zu kälterer Tageszeit oder über Nacht wieder erstarrte Schmelzfluß weiter belichtet, so entsteht eben aus der darin enthaltenen $\alpha$-Zimtsäure (fest) mehr $\alpha$-Truxillsäure, als wenn die ursprüngliche allo-Zimtsäure bei niederen Tagestemperaturen ohne intermediäre Verflüssigung belichtet worden wäre.

\section{Beschreibung der Versuche.}

\section{Zur Darstellung der allo-Zimtsäure').}

1. Durch Ilalbreduktion aus phenyl-propiolsaurem Natrium nach Paal und Hartmann ${ }^{2}$ ): A uf Empfeblung des Hrn. Geheimrat Paal, dem wir für seine freundliche Unterstützung und für die Überlassung seiner Apparate bestens danken, diente als Katalysator ein auf Bariumsulfat niedergeschlagenes, feinverteiltes Palladium. Die Reduktion verlief glatt und viel schneller, als nach den früher angegebenen Verfahren. Bei Anwendung von $0.1 \mathrm{~g}$ Palladium waren $15 \mathrm{~g}$ Phenyl-propiolsäure bereits in $90 \mathrm{Min}$., $24 \mathrm{~g}$ in $210 \mathrm{Min}$. reduziert. Zu ihrer Reinigung wird die allo Säure am besten mit Wasser, überschüssigem Bariumcarbonat und Tierkohle gekocht, dann die Salzlösung filtriert, angesāuert, die freie Säure ausgeäthert, der Extrakt im trocknen Luftstrom eingedunstet und der Rückstand aus Ligroin (Sdp. 60-70 ) umkrystallisiert.

2. Aus trans-Zimtsäure auf photochemischem Wege nach Störmer ${ }^{3}$ ).

Benzol-Lösung (3.5: 100) in Uviolglas-Röhren von $2-3 \mathrm{~cm}$ lichter Weite $100 \mathrm{Stdn}$. an Schottscher Uviollampe bestrahlt: Ausbeute $25-30 \%$ alloSäure (in Übereinstimmung mit Störmer).

Benzol-Lösung in Uviolglas-Kngeln von $500 \mathrm{cem}$ Inhalt $147 \mathrm{Stdn}$. an Quarz-Quecksilberlampe bestrahlt: Ausbeute $13 \%$ allo-Säure.

Benzol-Lösung in Uviolglas-Gefäßen 6-8 Wochen in direktem Somnenlicht : $10-17 \%$ allo.Säure.

1) Näheres bei Steinberger, Dissertat, Leipzig 1921.

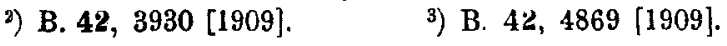


Die Aufarbeitung der Belichtungsprodukte wie oben. Zur Entfernung der bei den Lichtreaktionen entstandenen, teilweise recht erheblichen Mengen gelber, harziger Beimengungen wirden die Bariumsalze wiederholt mit Alkohol ausgekocht.

\section{Die quantitative Trennug der bei der Belichtung von} cis- und trans-Zimtsäure entstehenden Reaktionsprodukte.

Hierfür kamen in Betracht die Verfahren von de Jong') und Störmer ${ }^{2}$ ). Wir haben beide kombiniert und das Reaktionsgemisch zunäcbst mit einer hinreichenden Menge Benzol auf dem Wasserbad digeriert. Nachdem das Ganze einen Tag im Dunkeln gestanden batte, wurde filtriert. Die Lösung enthielt die Zimtsäuren, der ungelöste Anteil das Truxillsäure-Gemisch, das gewogen und auf seinen Schmelzpunkt untersucht wurde.

Die Trennung der $\alpha$-Truxill- von der $\beta$-Truxinsäure erfolgte nach de Jong Zur Trennung der Zimtsäuren wurden zwei Petroläther-Fraktionen (Sdp. $30-40^{\circ}$ u. Sdp. $60-70^{\circ}$ ) verwandt.

$$
\begin{aligned}
& 10 \mathrm{ccm}\left(\mathrm{Sdp} .30-40^{\circ}\right) \text { lüsen } 0.0025 \mathrm{~g} \text { trans-Säure }\left(10.5^{\circ}\right) \\
& 10 \text { - > }>\text { ca. } 0.20 » \text { allo- » } \\
& 10 \times\left(\mathrm{Sdp} .60-70^{\circ}\right) \gg 0.0048 \cdot \text { trans }-\left(10.5^{\circ}\right) \\
& 10 \gg, \quad \text { ca. } 0.85 \% \text { allo- }
\end{aligned}
$$

Man verdampit also die ursprüngliche Benzollösung zur Trockne, digeriert den Rïckstand mit einer der beiden Petroläther-Sorten, filtriert von der ungelösten trans-Säure ab und erbält nach dem Eindurasten des Filtrats die cis-Säure. Von dem durch Wägung für die cis Säure gefundenen Wert ist noch die im Petroläther mitgelöste geringe Menge trans-Zimtsäure in Abzug zu bringen und dieser Betrag dem für trans-Zimtsäure gefundenen zuzuzäblen. Etwaige Beimengungen vou Benzaldehyd, Benzoesäure, $\varepsilon$-Truxillsäure ${ }^{3}$ ) und $\mathrm{Harz}$ wurden meist nicht berücksichtigt.

Die analytische Bestimmung der dimeren und ibre Trennung von den monomeren Säuren ist recht genau. Dagegen krankt die quantitative Trennung der Monocarbonsäuren daran, daß der Petroläther bei niederer Temperatur nur schwer völlig verdunstet, also zu hohe Werte gefunden werden, während bei höherer Temperatur leicht Verluste durch Sublimation von Zimtsäure eintreten.

') R. 31, 258 [1912]. Ein Verfahren zur Trennung auch der anderen Lier zu vernachlässigenden Truxill- und Truxinsäuren s. C. 1919, III 1000.

2) B. 52, 1262 [1919] Ein anderes Verfahren ist beschrieben vou Störmer und Laage, B. 54, 80 [1921].

3) Störmer und Emme1, B. 53, 500 [1920]. 
Belichtungsversuche mit cis- und trang-Zimtsäure.

Belichtung flüssiger allo-Zimtsäure-Schmelze an der Quarzlampe:

1. $2 \mathrm{~g}$ allo-Sẵre in dauernd flüssigem Zustand $85 \mathrm{Stdn}$. belichtet. Lampenabstand $1-2 \mathrm{~cm}$. Starke Braunfärbung.

In $1.6222 \mathrm{~g}$ des Reaktionsprodukts gef. $0.5818 \mathrm{~g}$ cis-Săure und $1.0101 \mathrm{~g}$ trans-Săure (nebst Nebenprodukten). Truxillsäure war nicht entstanden.

Ergebnis: $35.86 \%$ cis-Säure und $64.12 \%$ trans Säure (die gelben Nebenprodukte eingerechnet).

2. $2.2 \mathrm{~g}$ allo-Säure in flüssigem Zustand $96 \mathrm{Stdn}$. belichtet. Starke Braunfärbung.

ln $1.9262 \mathrm{~g}$ des Reaktionsprodukts gef. $1.1508 \mathrm{~g}$ trans-Săure (einschließlich brauner Nebenprodukte); aus Petroläther (Sdp. 90-100 $0^{\circ}$ ) amkrystallisiert, Schmp. $130^{\circ}$. Truxillsäure war nicht entstanden.

Ergebnis: $40.3 \%$ cis.Säure, $59.7 \%$ trans. Säure.

\section{Belichtung von Zimtsäuren in fester Form.}

I. Versuche an der Quarz-Quecksilberlampe: 1 a $1.5 \mathrm{~g}$ allo-Zimtsäure, erstarrte Schmelze, in einer Petri-Schale 200 Stdn. belichtet. $6-7 \mathrm{~cm}$ Lampenabstand. Gelbfärbung.

In $0.6242 \mathrm{~g}$ des Reaktionsprodukts gef. $0.5966 \mathrm{~g}$ Monocarbonsäuren $(03922 \mathrm{~g}$ cis-Săure u. $0.2234 \mathrm{~g}$ trans-Sāure nebst Verunreinigungen, die wohl aus Benzoesäure u. harzigen Nebenprodukten bestanden), und $0.0276 \mathrm{~g}$ TruxillsăareGemisch (Schmp. 195-200\%).

$0.0248 \mathrm{~g}$ Truxillsäure-Gemiseh, in $10 \mathrm{ccm}$ Kalilauge, erforderten $7.86 \mathrm{~cm}$ Schwei̊elsăure; also gef.: $-\mathrm{g} a$-Truxillsăuro, $0.0297 \mathrm{~g} \beta$-Truxinsăure. (Der gefundene Mehrbetrag an $\beta$-Truxinsăure liegt innerbalb der Versuchsfehlergrenzen).

Ergebnis: $60,9 \%$ cis-Säure, $34.7 \%$ trans-Säure, $4.4 \% \beta$.Truxinsäure.

I, 1 b. 1 g allo- u. $1 \mathrm{~g}$ trans-Zimtsäure, Versuchsbedingungen wie oben. Gelbfärbung.

In $1.3326 \mathrm{~g}$ des Reaktionsprodukts gef. $1.2580 \mathrm{~g}$ Monocarbonsăuren. $(0.5609 \mathrm{gg}$ cis-Säure u. $0.7918 \mathrm{~g}$ trans-Säure nebst Verunreinigungen) u. $0.0746 \mathrm{~g}$ Truxillsäure-Gemisch (Schmp. 240-2710).

$0.0599 \mathrm{~g}$ Truxillsäure-Gemisch, in $10 \mathrm{ccm}$ Kalilange, erforderten $7.80 \mathrm{ccm}$ Schwefelsäure;"also gef. $00291 \mathrm{~g} \alpha$-Truxillsäure (Schmp. 272-2740) u. $0.0308 \mathrm{~g}$ $\beta$-Truxinsăure.

Ergebnis: $39.1 \%$ cis-Säure, $55.3 \%$ trans-Säure, $2.7 \% \alpha$-Truxillsäure, $2.9 \% \quad \beta$-Truxinsäure. 
$\mathrm{I}, 2.1 .5 \mathrm{~g}$ allo. u. $1.5 \mathrm{~g}$ trans-Zimtsäure, erstarrte Schmelze, in einer Petri-Schale $360 \mathrm{Stdn}$. belichtet. Gelbfärbung. Vorübergehende Verflüssigung während der Belichtung.

In $23175 \mathrm{~g}$ des Reaktionsprodukts gef. $2.1545 \mathrm{~g}$ Monocarbonsăuren $(0.1226 \mathrm{~g}$ cis-Säure u. $1.9174 \mathrm{~g}$ trans-Säure nebst Verunreinigungen) und $0.1630 \mathrm{~g}$ Truxillsäure-Gemisch (Sehmp. 258-264").

$0.1598 \mathrm{~g}$ Truxillsăure-Gemisch, in $20 \mathrm{ccm}$ Kalilauge, erforderten $17.8 \mathrm{~cm}$ Schwefelsäure; also gef. $0.1359 \mathrm{~g} \alpha$-Truxilloăure (Schmp. 274-2750), $0.0239 \mathrm{~g}$ $\beta$-Truxinzäure.

Ergebnis: $5.6 \%$ cis-Säure, $87.4 \%$ trans-Säure, $5.9 \% \alpha$-Truxillsäure, $1.1 \% \beta$-Truxinsäure.

I, 3. $0.8 \mathrm{~g}$ allo- u. $1.6 \mathrm{~g}$ trans-Zimtsäure, erstarrte Schmelze, in einer Petri-Schale 112 Stdn. belichtet. Gelbfärbung.

In $2.0863 \mathrm{~g}$ des Rohprodults gef. $2.0026 \mathrm{~g}$ Monocarbonsăuren $(0.3848 \mathrm{~g}$ (is-Săure u. $1.5828 \mathrm{~g}$ trans-Säure nebst Verunreinigungen) u. $0.0837 \mathrm{~g}$ Truxillsăure-Gemisch (Schmp. 274-275\%); also fast nur $\alpha$-Truxillsänre.

Ergebnis: $18.8 \%$ cis-Süure, $77.2 \%$ trans-Säure, $4 \% \alpha$-Truxillsãure.

I, 4a. $1.0012 \mathrm{~g}$ allo-Säure, feinverteilt durch Verdunsten der ätherischen Lösung in einem Quarzkolben. 364 Belichtungsstunden, in $20 \mathrm{~cm}$ Abstand über der Lampe hängend.

Gewicht des rein weißen Reaktionsprodukts $0.9944 \mathrm{~g}$. Darin gef. 0.9942 g Monocarbonsăure-Gemisch $(0.9338 \mathrm{~g}$ cis-Säuro u. $0.0152 \mathrm{~g}$ transSäure), keine Traxillsăuren.

Ergebnis: $97.7 \%$ cis-Säure, $1.6 \%$ trans-Säure, $0.7 \%$ Gewichtsverlust.

I, $4 \mathrm{~b} .1 .0140 \mathrm{~g}$ allo- u. $1.0010 \mathrm{~g}$ trans.Zimtsăure, Versuchsbedingungen wie oben.

Gewicht des reiu weißen Reaktionsprodults 2.0042 g. Darin gef. $1.9990 \mathrm{~g}$ Monocarbonsäure-Gemisch $(0.9616 \mathrm{~g}$ cis-Säure 'u. $1.0142 \mathrm{~g}$ transSäure) u. $0.0052 \mathrm{~g}$ Truxillsăuren.

Ergebnis: $48.3 \%$ cis-Säure, $50.9 \%$ trans-Säure, $0.3 \%$ Truxillsäuren, $0.5 \%$ Gewichtsverlust.

I, 4 c. $1.5838 \mathrm{~g}$ trans-Zimtsüure, Versuchsbedingungen wie oben.

Gewicht des rein weißen Reaktionsprodukts $1.5768 \mathrm{~g}$. Darin gef. $1.5458 \mathrm{~g}$

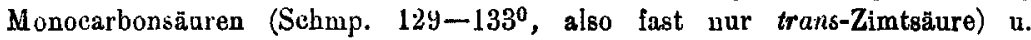
$0.0310 \mathrm{~g}$ Truxillsäure-Gemisch.

Ergebnis: $97.6 \%$ trans-Säure, $2 \%$ Truxillsäuren, $0.4 \%$ Sulstaazverlast. 
II. Versuche im Sonnenlicht.

Die Belichtung erfolgte zwischen zwei Glasplatten. Die Versuchsobjekte blieben fest und fast rein weiß.

II, 1 a. $5 \mathrm{~g}$ allo-Zimtsäure, pulverisiert. 14 Belichtungstage, meist trüb.

In $1.0491 \mathrm{~g}$ des Reaktionsprodukts gef. $0.8569 \mathrm{~g}$ Monocarbonsäuren $\left(0.4532 \mathrm{~g}\right.$ cis-S̈̈ure (Schmp. $\left.45-62^{\circ}\right)$ u. $0.3772 \mathrm{~g}$ trans-Säure (Schmp. $124-$ $\left.130^{\circ}\right)$ u. $0.1922 \mathrm{~g}$ Truxillsäure-Gemisch (Schmp. 19a-2070). Dieeses, mit Benzol gereinigt, schmolz bei $206-208^{\circ}$, war also reine $\beta$.Truxinsäure. $\alpha$-Truxillsäure war nicht nachweisbar.

Ergebnis: $446 \%$ cis-Säure, $37.1 \%$ trans-Säure, $18.3 \%$ $\beta$-Truxinsäure.

II, 1b. Nach weiteren 14 Belichtungstagen bei strablender Sonnenbeleuchtung:

In $1.0966 \mathrm{~g}$ des Reaktionsprodults gef. $0.3176 \mathrm{~g}$ Monocarbonsäure (0.2416 g trans-Säure, der Rest harziges Nebenprodukt) u. $0.7790 \mathrm{~g}$ Truxillsäure-Gemisch.

$0.2822 \mathrm{~g}$ Truxillsảure-Gemisch, in $20 \mathrm{cem}$ Kalilauge, erforderten $7.37 \mathrm{cem}$ Schwefelsäure, also gef. $0.0799 \mathrm{~g} \alpha$-Truxillsäure (Schmp. 274-2760) u. $0.2023 \mathrm{~g} \beta$-Truxinsäure (Schmp. $206-208^{\circ}$ ).

Ergebnis: $22 \%$ trans-Siure, $20.1 \% \quad \alpha$-Truxillsäure, $50.9 \%$ $\beta$-Truxinsäure, $7 \%$ Harz.

II, 2 a. $1 \mathrm{~g}$ allo Zimtsäure, pulverisiert. 17 Belichtungstage.

In $0.9649 \mathrm{~g}$ des Rohprodukts gef. $0.5213 \mathrm{~g}$ Monoharbonsäuren $(0.1528 \mathrm{~g}$ cis-Säure n. $0.4037 \mathrm{~g}$ trans-Säure (Schmp. $120-128^{\circ}$ ) u. $0.4436 \mathrm{~g}$ Truxillsăure Gemisch (Schmp. 204-2220).

$0.4374 \mathrm{~g}$ Trusillsäure-Gemisch, in $30 \mathrm{ccm}$ Kalilange, erforderten $11.15 \mathrm{cem}$ Schwefelsäure; also gef. $0.1356 \mathrm{~g} \alpha$-Truxillsäare (Schmp. 272-2730) u. 0.3018 g $\beta$-Truxinsäure (Schmp. 205-207\%).

Ergebnis: $14.8 \%$ cis.Säure, $39.2 \%$ trans-Säure, $14.3 \%$ $\alpha$-Truxillsäure, $31.7 \% \quad \beta$-Truxinsäure.

II, $2 \mathrm{~b}$. $1 / \mathrm{g}$ g allo- u. $1 / 2 \mathrm{~g}$ trans-Säure, Versuchsbedingungen wie oben.

In $0.8948 \mathrm{~g}$ des Reaktionsprodukts gef. $0.2410 \mathrm{~g}$ Monocarbonsäuren $\left\{0.0576 \mathrm{~g}\right.$ cis- und $0.1734 \mathrm{~g}$ trans-Sänre (Schmp. $\left.123-128^{\circ}\right)$ u. $0.6538 \mathrm{~g}$ Truxillsäure-Gemisch (Schmp. 206-260\%).

$0.6488 \mathrm{~g}$ Truxillsăure-Gemisch, in $50 \mathrm{ccm}$ Kalilange, erforderten $38.35 \mathrm{ccm}$ Schwefelsäure; also gef. $0.4838 \mathrm{~g} \alpha$-Truxillsăure (Schmp. $273-275^{\circ}$ ) u. $0.1650 \mathrm{~g} \beta$-Truxinsäure (Schmp. 205-2070).

Ergebnis: $6.7 \%$ cis-Säure, $20.2 \%$ trans-Säure, $54.5 \%$ $\alpha$-Truxillsäure, $18.6 \% \beta$-Truxinsäure.

IL, 3 a. 1 g allo-Säure, erstarrte Schmelze. 20 Belichtungstage. 
In $0.8299 \mathrm{~g}$ des Reaktionsprodukts gef. $0.3789 \mathrm{~g}$ Monocarbonsäuren $(0.1016 \mathrm{~g}$ cis-Säure u. $0.2762 \mathrm{~g}$ trans-Săure) u. $0.4510 \mathrm{~g}$ Truxillsäure-Gemisch (Schmp. 204-260\%).

$0.4476 \mathrm{~g}$ Truxillsäure-Gemisch, in $40 \mathrm{ccm}$ Kalilauge, erforderten $22.40 \mathrm{ccm}$ Schwefelsäure; also gef. $0.1741 \mathrm{~g} \alpha$-Truxillsăure $\mathfrak{a}$. $0.2735 \mathrm{~g} \beta$-Truxinsăure (Schmp. 206-207\%).

Ergebnis: $12.3 \%$ cis.Säure, $33.4 \%$ trans-Säure, $21.1 \%$ $\alpha$-Truxillsäure, $33.2 \beta$-Truxinsäure.

II, $3 \mathrm{~b}$. 1/a g allo- u. 1/2 g trans-Säure, Versuchsbedingungen wie oben.

In $08030 \mathrm{~g}$ des Reaktionsprodukts gef. $04173 \mathrm{~g}$ Monocarbonsäuren $\left(0.9982 \mathrm{~g}\right.$ cis-Sãure u. $0.3050 \mathrm{~g}$ trans-Säure (Schmp. $\left.\left.123-131^{\circ}\right)\right)$ u. $0.3857 \mathrm{~g}$ Truxillsäure-Gemisch (Schmp. 235-2700),

$0.3810 \mathrm{~g}$ Truxillsäare-Gemisch, in $30 \mathrm{ccm}$ Kalilauge, erforderten $20.48 \mathrm{ccm}$ Schwefelsäure; also gef. $0.23 \$ 8 \mathrm{~g} \alpha$-Truxillsäure $\mathfrak{u} .0 .1422 \mathrm{~g} \quad \beta$-Truxinsăure (Schmp. 206-2080).

Ergebnis: $12.7 \%$ cis-Süure, $39.3 \%$ trans-Säure, $30.1 \%$ $\alpha$-Truxillsäure, $179 \% \beta$-Truxinsäure.

II, 4 a. $1.5 \mathrm{~g}$ allo-Säure, erstarrte Schmelze. 27 Belichtungstage.

In $1.3926 \mathrm{~g}$. des Reaktionsprodukts gef. $0.8660 \mathrm{~g}$ Monocarbonsăuren $\left(0.1224 \mathrm{~g}\right.$ cis-Säure und $0.8100 \mathrm{~g}$ trans-Säure (Schmp. $\left.\left.120-130^{\circ}\right)\right)$ a. $0.5266 \mathrm{~g}$ Truxillsäure-Gemisch (Schmp. 203-208\%)

$0.4693 \mathrm{~g}$ Truxillsăure-Gemiseh, in $40 \mathrm{ccm}$ Kalilauge, erforderten $14.55 \mathrm{~cm}$ Schwefelsăure; also gef. $0.0615 \mathrm{~g} \alpha$-Truxillsäure (Schmp. 270-2740) a. $0.4078 \mathrm{~g} \beta$-Truxinsäure (Schmp. 206-2070).

Ergebnis: $8.2 \%$ cis Säure, $54 \%$ trans-Säure, $4.9 \%$-Truxillsäure, $32.9 \% \beta$ Truxinsäure.

II, $4 \mathrm{~b}$. $1 \mathrm{~g}$ allo- u. $1 \mathrm{~g}$ trans-Säure, Versuchsbedingungen wie iben.

In $1.5832 \mathrm{~g}$ des Reaktionsprodukts gef. $0.8996 \mathrm{~g}$ Monocarbonsäuren ( $0.1290 \mathrm{~g}$ cis-Säure u. $0.7730 \mathrm{~g}$ trans-Säure) u. $0.6836 \mathrm{~g}$ Truzillsăure-Gemisch (Schmp. $220-260^{\circ}$ ).

$0.6796 \mathrm{~g}$ Truxillsäure-Gemisch, in $50 \mathrm{ccm}$ Kalilauge, erforderten $36.10 \mathrm{cem}$ Schwefelsăure; also gef. $0.4761 \mathrm{~g} \alpha$-Truxillsäure und $0.2035 \mathrm{~g} \beta$-Truxinsäure (Schmp. 204-207\%).

Ergebnis: $8.1 \%$ cis-Säure, $48.7 \%$ trans.Säure, $\quad 30.3 \%$ $\alpha$-Truxillsäure, $12.9 \% \beta$-Truxinsäure.

II, 5 a. $1.5 \mathrm{~g}$ allo Säure, pulverisiert. 27 Belichtungstage.

In $1.4281 \mathrm{~g}$ des Reaktionsprodukts gef. $0.5860 \mathrm{~g}$ Monocarbonsäuren $(0.1030 \mathrm{~g}$ cis-Săure a. $0.5570 \mathrm{~g}$ trans-Săure) u. $0.6836 \mathrm{~g}$ Truxillsäure-Gemisch (Schmp. 205-207\%). 
$0.8350 \mathrm{~g}$ Truxillaäure-Gemisch, in $60 \mathrm{ccm}$ Kalilauge, erforderten $15.93 \mathrm{ccm}$ Schwefelsäure; also gef. $0.1226 \mathrm{~g} \alpha$-Truxillsăure $\mathfrak{u} .0 .7124 \mathrm{~g} \beta$-Truxinsäure (Schmp. 206-2070).

Ergebois: $6.4 \%$ cis-Säure, $34.6 \%$ trans-Säure, $8.7 \%$-Truxillsäure, $503 \%$ - 3 -Truxinsäure.

II, $5 \mathrm{~b} .1 \mathrm{~g}$ allo- $\mathrm{u}, 1 \mathrm{~g}$ trans-Säure, pulverisiert. Versucbsbedingungen wie oben.

In $1.7548 \mathrm{~g}$ des Reaktionsprodukts gef, $0.4848 \mathrm{~g}$ Monocarbonsāuren $(0.0636 \mathrm{~g}$ cỉ-Säure u. $0.4398 \mathrm{~g}$ trans-Säure) $1.1 .2700 \mathrm{~g}$ Truxillsäure-Gemisch (Schmp. 220-260')

$1.2622 \mathrm{~g}$ Truxillsäure-Gemisch, in $90 \mathrm{~cm}$ Kalilauge, erforderten $70.10 \mathrm{~cm}$ Sehwefelsüure; also gef. $0.9835 \mathrm{~g} \alpha$-Truxillsäure u. $0.2787 \mathrm{~g} \quad \beta$-Truxinsüure (Schmp. 204-2070).

Ergebnis: $3.5 \%$ cis-Säure, $24.1 \%$ trans-Säure, $56.4 \% \alpha$-Truxillsäure, $16 \% \quad \beta$-Truxinsäure.

II, $6.3 \mathrm{~g}$ trans-Zimtsäure, pulverisiert. 23 Belichtungstage.

In $2.5866 \mathrm{~g}$ des Reaktionsprodukts gef. $0.3692 \mathrm{~g}$ Monocarbonsäuren und $2.2174 \mathrm{~g}$ Truxillsäure, die bei $267-275^{\circ} \mathrm{schmolz}$, also in der Hauptsache $a$-Truxillsäure war.

Das Monocarbonsäure-Gemisch, mit Ligroin ausgezogen, schmolz bei $132-133^{\circ}$, war also trans-Zimtsäure, In das Ligroin waren nur $0.0185 \mathrm{~g}$ rom

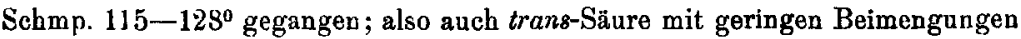
(cis-Säure?).

Ergebnis: $14.3 \%$ trans-Säure, $85.7 \% \quad \alpha$-Truxillsäure (ohve Berücksichtigung der Nebenprodukte).

Belichtung salzsaurer Suspensionen im Sonnenlicht.

Die Versuchsobjekte wurden in $14 \mathrm{ccm} 2-n$. Natronlauge gelöst, mit $4 \mathrm{ccm}$ konz. Salzsäure gefällt und mit $16 \mathrm{~cm}$ Wasser versetzt. Die Belichtung erfolgte in Glaskolben gleicher Beschaffenheit.

a) $1 \mathrm{~g}$ allo-Säure. 9 Belichtungstage. Benzaldehyd-Geruch.

Filterrückstand (weiß): $0.8904 \mathrm{~g}$. Aus dem salzsauren Filtrat durch Ausäthern erhalten: $0.0768 \mathrm{~g}$ harziges Nebenprodukt.

Im Filterrückstand gef. $0.5210 \mathrm{~g}$ trans-Zimtsāure (mit Ligroin grereinigt,

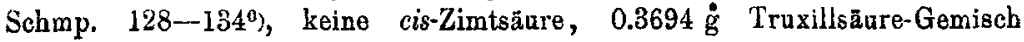
(Schmp. 200-2030).

$0.2596 \mathrm{~g}$ Truxillsäure-Gemisch, in $20 \mathrm{ccm}$ Kalilauge, erforderten $433 \mathrm{ccm}$ Schwefelsäure; also gef. $0.2542 \mathrm{~g} \beta$-Truxinsäure (Schmp. 205-208 ); da in der titrierten Flüssigkeit $\alpha$-Truxillsäure nicht nachzuweisen war, war wohl nur $\beta$-Truxinsäure vorhanden.

Ergebnis: $53.9 \%$ trans-Säure, $38.2 \% \quad \beta$-'Truxinsäure, $7.9 \%$ Harz. 
$1 \mathrm{~b} .1 / 2 \mathrm{~g}$ allo- und $1 / 2 \mathrm{~g}$ trans-Säure. Versuchsbedingungen wie oben. Benzaldehyd-Geruch.

Filterrückstand (weiß): $0.8998 \mathrm{~g}$. Aus dem salzsauren Filtrat durch Ausäthern erhalten: $0.0482 \mathrm{~g}$ harziges Nebenprodukt.

Im Filterrückstand gef. $0.5536 \mathrm{~g}$ trans-Zimtsänre (mit Ligroin gereinigt, Schmp. $128-133^{0}$ ), keine cis-Zimtsäure, $0.3462 \mathrm{~g}$ Truxillsäure-Gemisch (Schmp. 240-265\%).

$0.3390 \mathrm{~g}$ Truxillsäure-Gemisch, in $35 \mathrm{ccm}$ Kalilauge, erforderten $23.60 \mathrm{ccm}$ Schwefelsäure; also gef. $0.1680 \mathrm{~g} \alpha$-Truxillsäure u. $0.1710 \mathrm{~g} \quad \beta$-Truxinsäure (Schmp. 206-207\%).

Ergebnis: $58.4 \%$ trans-Säure, $18.1 \%$-Truxillsäure, $18.4 \%$ $\beta$-Truxinsäure, $5.1 \%$ Harz.

2 a. $1 \mathrm{~g}$ allo-Säure. 20 Belichtungstage. Benzaldehyd-Geruch.

Filterrückstand (schwach gelblich): $0.8410 \mathrm{~g}$. Aus dem salzsaurem Filtrat durch Ausäthern erhalten: $0.0732 \mathrm{~g}$ harziges Nebenprodukt.

Im Filterrückstand gef. $0.4894 \mathrm{~g}$ trans-Zimtsäure (wohl mit etwas Benzoesüure; die trans-Säure, mit Ligroin gereinigt und aus Wasser umkrystallisiert, schmolz bei $130-134^{\circ}$ und reduzierte sodaalkalisches Permanganat); keine cis-Zimtsäure, $0.3516 \mathrm{~g}$ Truxillsäure-Gemisch (Schmp. 204-208\%).

$0.3470 \mathrm{~g}$ Truxillsäure-Gemisch, in $30 \mathrm{ccm}$ Kalilauge, erforderten $8.68 \mathrm{ccm}$ Schwefelsäure; also gef. $0.0030 \mathrm{~g} \alpha$-Truxillsäure (Schmp. $270-274^{0}$ ) u. $0.3440 \mathrm{~g} \beta$-Truxinsäure (Schmp. 206-2080).

Ergebnis: $53.53 \%$ trans-Säure, 0.33\% $\alpha$-Truxillsäure, $38.13 \%$ $\beta$-Truxinsäure, $8.01 \%$ Harz.

2b. $1 / 2$ g allo- u. $1 / 2$ g trans-Säure. Versuchsbedingungen wie oben. Benzaldehyd-Geruch.

Filterrückstand (schwach gelblich): $0.8976 \mathrm{~g}$. Aus dem salzsauren Filtrat durch Ausāthern erhalten: $0.0674 \mathrm{~g}$ harziges Nebenprodukt.

Im Filterrückstand gef. $0.5366 \mathrm{~g}$ trans-Zimtsäure (wohl mit etwas Benzoesäure), keine cis-Zimtsãure, $0.3610 \mathrm{~g}$ Truxillsäure-Gemisch (Schmp. 230-260\%).

$0.3574 \mathrm{~g}$ Truxillsäure-Gemisch, in $30 \mathrm{ccm}$ Kalilauge, erforderten $20.00 \mathrm{ccm}$ Schwefelsäure; also gef. $0.2069 \mathrm{~g} a$-Truxillsäure (Schmp. 274-2750) u. $0.1505 \mathrm{~g} \beta$-Truxinsäure (Schmp. 206-2070).

Ergebnis: $55.6 \%$ trans.Säure, $21.7 \%$ ↔-Truxillsäure, $15.7 \%$ $\beta$-Truxinsãure, $7 \%$, Harz.

Die Untersuchung wird fortgesetzt. 